\title{
A!
}

This is an electronic reprint of the original article.

This reprint may differ from the original in pagination and typographic detail.

Kinnunen, J.; Törmä, P.; Pekola, J. P.

\section{Measuring charge-based quantum bits by a superconducting single-electron transistor}

Published in:

Physical Review B

DOI:

10.1103/PhysRevB.68.020506

Published: 30/07/2003

Document Version

Publisher's PDF, also known as Version of record

Please cite the original version:

Kinnunen, J., Törmä, P., \& Pekola, J. P. (2003). Measuring charge-based quantum bits by a superconducting single-electron transistor. Physical Review B, 68(2), 1-4. [020506]. https://doi.org/10.1103/PhysRevB.68.020506

This material is protected by copyright and other intellectual property rights, and duplication or sale of all or part of any of the repository collections is not permitted, except that material may be duplicated by you for your research use or educational purposes in electronic or print form. You must obtain permission for any other use. Electronic or print copies may not be offered, whether for sale or otherwise to anyone who is not an authorised user. 


\title{
Measuring charge-based quantum bits by a superconducting single-electron transistor
}

\author{
J. Kinnunen and P. Törmä \\ Department of Physics, P.O. Box 35 (YFL), FIN-40014, University of Jyväskylä, Finland
}

J. P. Pekola

Low Temperature Laboratory, Helsinki University of Technology, P.O. Box 3500, 02015 HUT, Finland

(Received 3 June 2003; published 30 July 2003)

\begin{abstract}
Single-electron transistors have been proposed to be used as a read-out device for Cooper pair charge qubits. Here we show that a coupled superconducting transistor at a threshold voltage is much more effective in measuring the state of a qubit than a normal-metal transistor at the same voltage range. The effect of the superconducting gap is to almost completely block the current through the transistor when the qubit is in the logical state 1, compared to the mere diminishment of the current in the normal-metal case. The time evolution of the system is solved when the measuring device is driven out of equilibrium, the effect of higher-order contributions is examined and the setting is analyzed numerically for parameters accessible by lithographic aluminum structures.
\end{abstract}

DOI: 10.1103/PhysRevB.68.020506

PACS number(s): 85.35.Gv

Nanoscale devices, such as Cooper pair boxes or coupled quantum dots, have been suggested as scalable and integrable realizations of quantum bits. The two logical states of a qubit are the different charge states, or in the case of a flux qubit, flux states of the system. There are several proposals for quantum gates ${ }^{1,2}$ and for interqubit couplings, ${ }^{3}$ as well as for measuring devices. ${ }^{4-7}$ Permanently coupled normalmetal transistors have been suggested as a device for measuring the state of a Cooper pair charge qubit. ${ }^{8,9}$ Also, a superconducting single-electron transistor (SET) in the Josephson current regime (low biasing voltage) has been experimentally tested. ${ }^{10}$ In this work, we show that in the regime of high biasing voltages, the superconducting SET ${ }^{11-13}$ leads to a highly efficient quantum nondemolition measurement ${ }^{14}$ due to the blocking effect of the gap.

The setting is shown in Fig. 1. In the upper part, the Cooper pair box forms the qubit, its state characterized by the number of excess Cooper pairs in the box $n$. In the lower part, the superconducting single-electron transistor is capacitively coupled to the qubit with its state characterized by the excess charge on the island $e N$. In addition, the quantum number $m$ counts the number of charges passing through the SET in left-to-right direction. Without a biasing voltage $V_{\text {bias }}$ across the SET, there is no dissipative current and no information is received. Moreover, in the absence of a dissipative environment, no dephasing of the composite system will occur and quantum operations on the qubit can be performed.

In order to perform the measurement, a biasing voltage is applied. As different qubit eigenstates correspond to different conductance in the SET, by observing the current, one receives information on the state of the qubit. The time needed for the current to give the essential information is called the measurement time. The back action caused by the SET dephases the qubit, and eventually also destroys the logical state of the qubit. The corresponding time scales are called the dephasing and the mixing times, respectively. For a good nondemolition measurement of the logical state $\left(|a|^{2}\right.$ and $|b|^{2}$ in $a|0\rangle+b|1\rangle$ ), one expects to have a much longer mix- ing than a measurement time scale. We show that for a superconducting SET, this is the case.

The total Hamiltonian ${ }^{8}$ consists of three parts: the Hamiltonians of the SET, the qubit, and the interaction $H_{\mathrm{set}}, H_{\mathrm{qb}}$, and $H_{\text {int }}$, respectively. The SET Hamiltonian is defined as

$$
H_{\text {set }}=E_{\text {set }}\left(N-Q_{\text {set }}\right)^{2}+H_{\mathrm{T}}+H_{\mathrm{L}}+H_{\mathrm{R}}+H_{\mathrm{I}},
$$

where $E_{\text {set }}$ is the charging energy, $Q_{\text {set }}$ the gate charge of the transistor, and

$$
H_{\mathrm{T}}=\sum_{k k^{\prime} \sigma} T_{k k^{\prime} \sigma}^{\mathrm{L}} c_{k \sigma}^{\dagger \mathrm{I}} c_{k^{\prime} \sigma}^{\mathrm{L}} e^{i \phi}+T_{k k^{\prime} \sigma}^{\mathrm{R}} c_{k \sigma}^{\dagger \mathrm{R}} c_{k^{\prime} \sigma}^{\mathrm{I}} e^{-i \phi} e^{i \Psi}+\text { H.c. }
$$

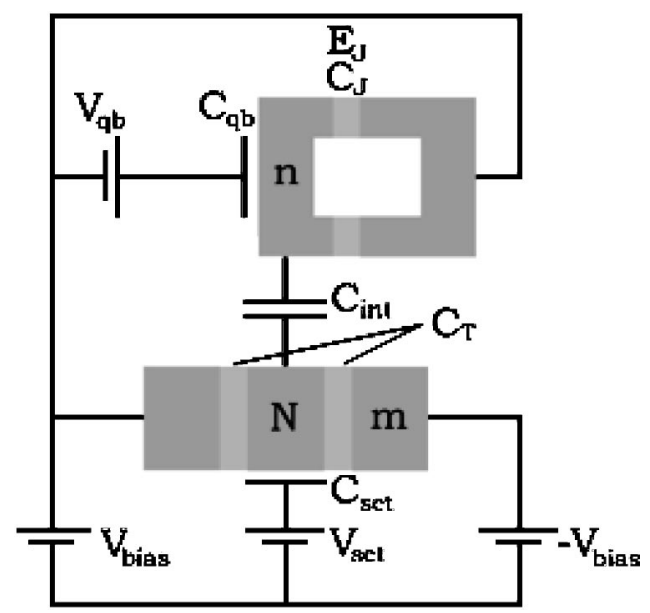

FIG. 1. A Cooper pair charge qubit is capacitively coupled to a measuring single-electron transistor. The quantum numbers $n, N$, and $m$ are explained in the text. The SET is symmetrically biased with voltage $V_{\text {bias }}$, and the voltage $V_{\text {set }}$ is the gate voltage. The energy scales are determined by the interaction capacitance $C_{\text {int }}$, the tunnel junction's capacitances $C_{\mathrm{T}}$, and the gate capacitance $C_{\text {set }}$. 
describes quasiparticle tunneling within the SET. The phase differences $\phi$ and $\Psi$ are the conjugate variables of $N$ and $m$, respectively. Thus, the operator $e^{i \phi}\left(e^{i \Psi}\right)$ corresponds to quasiparticle tunneling onto the island (right electrode) of the SET, increasing the quantum number $N(m)$ by one. The last three terms in Eq. (1) are defined as $H_{r}$ $=\Sigma_{k \sigma} \epsilon_{k \sigma} c_{k \sigma}^{\dagger r} c_{k \sigma}^{r},(r=\mathrm{L}, \mathrm{R}, \mathrm{I})$, where $\sigma$ labels the transverse channels including the spin and $k$ labels the wave vector within one channel. These describe the noninteracting electrons in the left electrode, the right electrode, and the island, respectively. The Cooper pair tunneling is excluded from the SET Hamiltonian due to the high biasing voltage. However, this approximation is not valid for a low voltage, and the contribution from the Cooper pair tunneling should be added when analyzing the effect of the SET on the qubit during logical operations.

Using the two-state approximation, the qubit Hamiltonian can be written as ${ }^{1}$

$$
H_{\mathrm{qb}}=-\frac{1}{2} \Delta E \sigma_{z}
$$

where the energy $\Delta E=\sqrt{\left[E_{\mathrm{qb}}\left(1-2 Q_{\mathrm{qb}}\right)\right]^{2}+E_{\mathrm{J}}^{2}}$. The interaction Hamiltonian describes the Coulomb interaction between the qubit and the transistor and is defined as

$$
H_{\text {int }}=E_{\text {int }} n N,
$$

where $E_{\text {int }}$ is the charging energy. All charging energies $E_{\text {int }}, E_{\text {set }}, E_{\mathrm{qb}}$ and gate charges $Q_{\mathrm{qb}}, Q_{\mathrm{set}}$ are determined by the capacitances and voltages of the system as given in Ref. 1. By rearranging the operators, the final form for the total Hamiltonian can be written as $H=H_{0}+H_{\mathrm{T}}$, where $H_{0}$ $=H_{\mathrm{L}}+H_{\mathrm{R}}+H_{\mathrm{I}}+H_{\mathrm{qb}}+E_{\mathrm{set}}\left(N-Q_{\mathrm{set}}\right)^{2}+H_{\text {int }}$.

We analyze the measurement process by master equation techniques. The master equation for the system reads

$$
\frac{\partial \sigma(t)}{\partial t}+\frac{i}{\hbar}\left[H_{0}, \sigma(t)\right]=\operatorname{Tr}_{\mathrm{L}, \mathrm{R}, \mathrm{I}} \int_{0}^{t} \Sigma\left(t-t^{\prime}\right) \sigma\left(t^{\prime}\right) d t^{\prime},
$$

where the trace is taken over the microscopic degrees of freedom of the transistor's left and right electrodes and the island. The elements of the transition matrix $\Sigma\left(t-t^{\prime}\right)$ $=-\left(1 / \hbar^{2}\right)\left(\left[V, U\left(t-t^{\prime}\right)[V, \cdot] U\left(t^{\prime}-t\right)\right]\right)$ are calculated by using the diagrammatic technique developed in Refs. 15 and 3 and the superconducting density of states $N(x)$ $=\left(|x| / \sqrt{x^{2}-\Delta^{2}}\right) N_{0}$, for $|x|>\Delta$. In zero-temperature limit, these elements are given by the so-called Basset function of first order, ${ }^{17}$ which must be analyzed numerically.

By performing the Laplace transform on master equation (5), the right-hand side becomes $\Sigma(s) \sigma(s)$. Assuming the density matrix $\sigma$ to change slowly in a time scale of $\hbar / E$ (typically of order $10^{-12} \mathrm{~s}$ ), the calculations can be restricted to the regime $s \ll E$. In the normal-metal case, $\Sigma(s)$ varies only slowly as a function of small $s$, and therefore, the zeroth-order approximation is reasonably good. However, when the energy $E / 2$ is close to the gap energy $\Delta, \Sigma(s)$ has a strong $s$ dependence which is approximately linear for small $s$, as shown by Fig. 2. Using the linear approximation $\Sigma(s)=\Sigma\left(s_{0}\right)+b\left(s-s_{0}\right)$ and performing the inverse Laplace

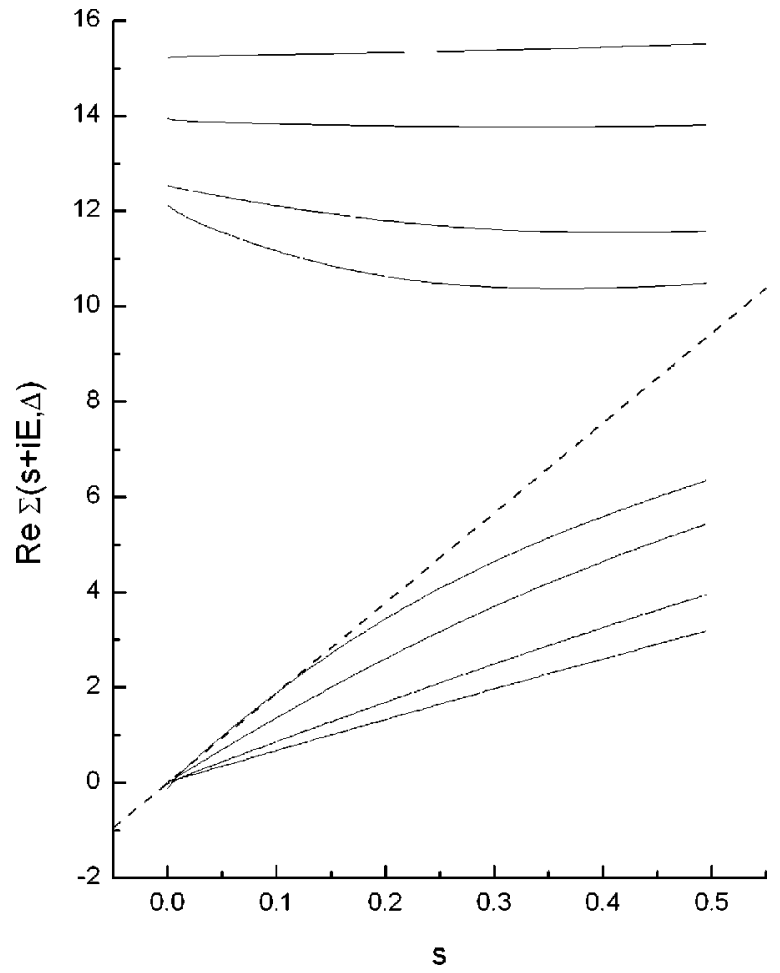

FIG. 2. The complex transition coefficient $\Sigma(s+i E)$ plotted as a function of $s$ with fixed $\Delta=2.3$ for different values of the energy $E$. The real components are drawn in solid curves and the imaginary parts in dotted ones. The corresponding energy values are in the order from the topmost curve to the lowest curve: $E=3.4, E$ $=3.8, E=4.2, E=4.35, E=4.85, E=5.0, E=5.4$, and $E=5.8$. For small values of $s$, the real components are nearly linear and the linear approximation discussed in the text is shown in a dashed line for the curve $E=4.35$.

transform, the right-hand side becomes $(\Gamma+\Lambda \partial / \partial t) \sigma(t)$. The coefficients $\Gamma$ and $\Lambda$ depend on the bias voltage and the energies of the system. Moving all the derivatives of the density matrix to the left-hand side gives a master equation

$$
(1-\Lambda) \frac{\partial \sigma(t)}{\partial t}+\frac{i}{\hbar}\left[\sigma(t), H_{0}\right]=\Gamma \sigma(t)
$$

where $\Gamma$ and $\Lambda$ are tridiagonal matrices consisting of the zeroth- and first-order terms of the $\Sigma(s)$ matrix, respectively. To guarantee the existence of the inverse of $(1-\Lambda)$, the elements of the $\Lambda$ coefficient are required to be small. This requirement is fulfilled when the tunneling rate within the SET is small (that is, $\left|T^{\mathrm{L}}\right|,\left|T^{\mathrm{R}}\right| \ll 1$ ). Multiplying Eq. (6) from the left by $(1-\Lambda)^{-1}$, the final form of the master equation is obtained as

$$
\frac{\partial \sigma(t)}{\partial t}=(1-\Lambda)^{-1}\left[-\frac{i}{\hbar}\left[\sigma(t), H_{0}\right]+\Gamma \sigma(t)\right]=\widetilde{\Gamma} \sigma(t)
$$

The elements of the $\Sigma(s)$ matrix are analyzed by explicitly writing the Laplace transform as 


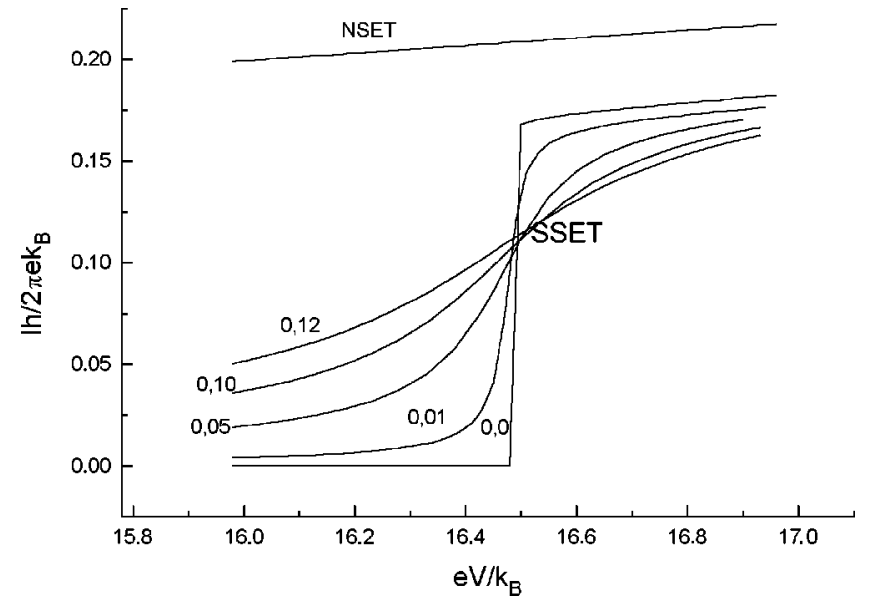

FIG. 3. The IV-curves of SSET using the linear approximation $\Sigma(s)=\Sigma\left(s_{0}\right)+b\left(s-s_{0}\right)$ for the transition matrix. Higher-order processes are simulated by varying $s_{0}$. The topmost curve is for NSET and the following ones are for SSET with $s_{0}=0.12, s_{0}$ $=0.10, s_{0}=0.05, s_{0}=0.01$, and $s_{0}=0.0$, out of which $s_{0}=0.10$ gives differential conductance at the threshold close to $e^{2} / 2 \pi \hbar$.

$$
\Sigma(s)=\int_{0}^{\infty} \Sigma(t) e^{-s t} d t=\int_{0}^{\infty} F(t)^{2} e^{(-s+i E) t} d t .
$$

The coefficient $b$ for the linear approximation is determined by calculating $\Sigma(s)$ for two values of $s=0.0$ and $s=0.1$ and fitting a line. Approximation is valid for values of $s$ up to 0.25 as shown in Fig. 2, corresponding to a time scale of $10^{-11} \mathrm{~s}$. The zero-order term $\Gamma$ describes sequential tunneling in the transistor and the first-order term $\Lambda$ contains some higher-order processes.

Doing the linear approximation $\Sigma(s)=\Sigma\left(s_{0}=0.0\right)+b s$ gives zero current in the Coulomb blockade regime: zeros in the transition matrix $\Gamma$ smear out the higher-order corrections. However, by using a nonzero value of $s_{0}$, one obtains small transition probabilities in $\Gamma$ matrix and nonvanishing current even in the Coulomb blockade regime. This allows one to estimate approximately the effect of higher-order contributions by varying $s_{0}$. The IV-curves corresponding to different choices of parameter $s_{0}$ are shown in Fig. 3, showing rounding of the steplike behavior, and for a suitable choice of $s_{0}$, one obtains the differential conductance $e^{2} / 2 \pi \hbar$ at the threshold, which are observed in Ref. 12.

Varying the parameter $s_{0}$ on a small range $[0.0,0.15]$ has a rather small effect on time scales (measurement time $t_{\mathrm{ms}}$ varies on range $\left[1.0 \times 10^{-10} \mathrm{~s}, 2.4 \times 10^{-10} \mathrm{~s}\right]$, while mixing time $t_{\text {mix }}$ of $1 \times 10^{-8} \mathrm{~s}$ is unaffected), so one can argue that the effect of the higher-order contributions is small. This can be understood by observing that for our choice of interaction energy $E_{\text {int }}=0.50 \mathrm{~K}$, the rounding has only small effect on the two values of the conductivity. For $E_{\text {int }} \rightarrow 0$, the effect of higher-order contributions is increased. However, one should notice that the above treatment becomes increasingly accurate as $E_{\text {int }}$ approaches zero as the parameter $s_{0}$ is determined using the differential conductance at the threshold.

Elements of the matrix $\widetilde{\Gamma}$ describing the transitions within the qubit are proportional to a small mixing angle $\epsilon$ defined by $\tan \epsilon=E_{\text {int }} \sin \eta /\left(\Delta E+E_{\text {int }} \cos \eta\right)$, where the angle $\eta$ is defined as $\tan \eta=E_{\mathrm{J}} /\left[E_{\mathrm{qb}}\left(1-2 Q_{\mathrm{qb}}\right)\right]$. By approximating these elements by zeros, the matrix $\widetilde{\Gamma}$ separates into four parts: one part describing the system when the qubit is in (diagonal) state 00 , one part when the qubit is in state 11 , and two parts for the nondiagonal qubit states 01 and 10. The first two parts describe slowly damping conductance peaks that propagate in time. They give raise to the measurement, and the measurement time is defined as the time when the width of the peaks is smaller than the distance between their centers. For a detailed description see Ref. 1. The coherence of the qubit is described by the strength of the nondiagonal elements, and thus the rate at which the nondiagonal elements vanish gives the dephasing time. For the mixing time, one has to include the small mixing elements proportional to $\epsilon$ and one obtains eight eigenvalues of the transition matrix. Four of the eigenvalues describe the dephasing, two describe the measurement, and one eigenvalue is zero (describing the trace preserving symmetry). The remaining real eigenvalue $\lambda_{\text {mix }}$ gives the mixing time as $t_{\text {mix }}=1 / \lambda_{\text {mix }}$.

If the mixing time is very long compared to the measurement time, the measuring device disturbs the probability amplitudes of the qubit $\left(|a|^{2}\right.$ and $|b|^{2}$ in $\left.a|0\rangle+b|1\rangle\right)$ only a little. The uncertainties of the charge $\Delta Q$ and its conjugate variable (phase or flux) $\Delta \Phi$ are linked to each other by the uncertainty principle $\Delta Q \Delta \Phi \geqslant \hbar / 2$. According to this principle, if the precision of the charge measurement is very high, the phase becomes completely undetermined. The information that can be gathered from the SET contains no information of the phase, and thus the precision of measuring the charge within the qubit $\left(|a|^{2}\right.$ and $\left.|b|^{2}\right)$ can be very high.

The time scales are calculated for a specific set of parameters including the higher-order effects by doing the linear approximation with $s_{0}=0.10$ as explained above. With aluminum structures in mind, the superconducting gap of the SET is chosen to be $\Delta=2.3 \mathrm{~K}$ and the charging energies $E_{\mathrm{qb}} \approx 1.0 \mathrm{~K}, E_{\mathrm{set}} \approx 5.2 \mathrm{~K}$ and $E_{\mathrm{int}} \approx 0.50 \mathrm{~K}$. The gate charges and tunneling coefficients are chosen as $Q_{\mathrm{qb}}=0.35, Q_{\text {set }}$ $=0.15,\left|T^{L}\right|^{2}=\left|T^{R}\right|^{2}=0.01$. To justify the charge-qubit approximation $E_{\mathrm{qb}} \gg E_{\mathrm{J}}$, the strength of the Josephson coupling is chosen as $E_{\mathrm{J}}=0.10 \mathrm{~K}$. Finally, the biasing voltage is chosen as $e V=16.5 \mathrm{~K}$. This set of values gives the measurement time $t_{\mathrm{ms}} \approx 2.0 \times 10^{-10} \mathrm{~s}$, the dephasing time $t_{\phi} \approx 1.1$ $\times 10^{-10} \mathrm{~s}$, and the mixing time $t_{\mathrm{mix}} \approx 1.3 \times 10^{-8} \mathrm{~s}$. The ratio between the mixing and the measurement times is high, i.e., $t_{\text {mix }} / t_{\text {ms }} \approx 65$, in agreement with the results in Ref. 6 . Though the measurement times of order $10^{-10} \mathrm{~s}$ are beyond the reach of actual measuring devices, the short measurement time shows that the SSET is capable of distinguishing the two qubit states even if, in practice, one is incapable of distinguishing between the two values of current quickly. On the other hand, the mixing time of order $10^{-8} \mathrm{~s}$ well exceeds the time scales of typical current electrometers $10^{-9} \mathrm{~s}$. For NSET, the same set of parameters gives $t_{\mathrm{ms}} \approx 6.0 \times 10^{-8} \mathrm{~s}$, $t_{\phi} \approx 1.0 \times 10^{-10} \mathrm{~s}$, and $t_{\mathrm{mix}} \approx 1.0 \times 10^{-8} \mathrm{~s}$. Our approach cannot be used for the NSET at the Coulomb blockade threshold, but this region has already been studied in Ref. 9. However, low voltage slows the measurement process, and the 


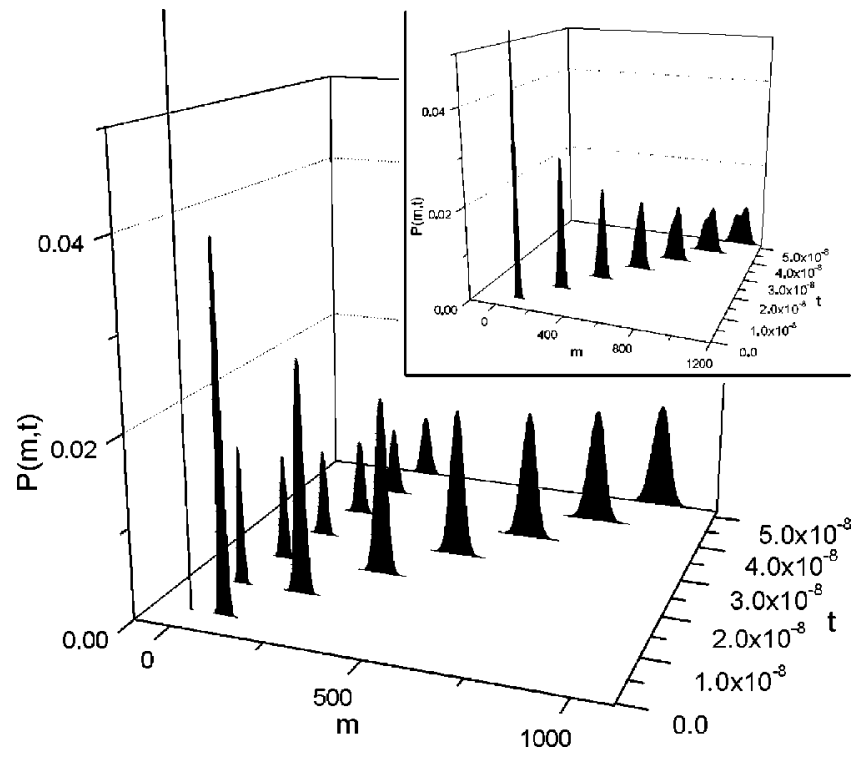

FIG. 4. The probability $P(m, t)$ that $m$ electrons have tunneled in a superconducting SET plotted as a function of time $t$ for initial amplitudes $\sqrt{0.75}(n=0)$ and $\sqrt{0.25}(n=1)$. The parameters are given in the text. The graph shows the separation of the two peaks, the faster corresponding to the qubit's state 0 and the slower to the state 1 . The two peaks are clearly distinguishable from each other, and thus the measurement time is very short. The curve in the box shows the corresponding evolution for normal-metal NSET.

excluded effect of environment may become significant, making the measurement more difficult.

The system has been simulated numerically by solving Eq. (7) using the Euler's method for the above parameter values. The probabilities $P(m, t)$ for $m$ electrons having been tunneled during time $t$ are plotted for the superconducting and normal-metal cases in Fig. 4. For SSET, the measure- ment time is very short, as the other peak (corresponding to the qubit state $n=1$ ) propagates in time very slowly. The mixing effects are small, as the peaks remain separated and the small visible spreading is caused mainly by the shot noise. The definition of the mixing time is very conservative, as the peaks are clearly distinguishable in SSET well beyond the mixing time calculated from the eigenvalues. The time taken for significant transitions to occur in the qubit is a longer order of magnitude (i.e., $10^{-7} \mathrm{~s}$ ). For NSET the separation of the peaks is not clear and it is, therefore, unlikely to be a sensitive enough quantum measurement device at the parameter range used above. Using niobium for the qubit allows one to use higher charging energy $E_{\mathrm{qb}}$ improving the charge-qubit approximation $E_{J} \ll E_{\mathrm{qb}}$. This has the effect of increasing the mixing time by more than order of magnitude without affecting other timescales.

To understand the higher signal-to-noise ratio of the SSET, note that for NSET the currents $I_{0}$ and $I_{1}$ corresponding to different qubit states are determined by $E_{\text {int }}$, which leads to small difference $I_{0}-I_{1}$. On the contrary, the sharp-

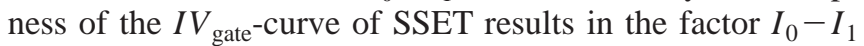
being almost independent of the interaction energy and relatively large. Thus one can achieve short measurement times (cf. Ref. 18). Even the small rounding of the steplike behavior caused by higher-order contributions does not significantly affect this picture. The mixing times for NSET and SSET are nearly equal because the back-action processes do not differ qualitatively. Therefore, signal-to-noise ratio for SSET becomes considerably higher than that for NSET.

This work was supported by the Academy of Finland (project 53903) under the Finnish Center of Excellence Project 2000-2005 (Project No. 44875, Nuclear and Condensed Matter Programme at JYFL). We thank M. Aunola for useful discussions.
${ }^{1}$ Y. Makhlin et al., Rev. Mod. Phys. 73, 357 (2001).

${ }^{2}$ D.V. Averin, Solid State Commun. 105, 659 (1998); quant-ph/9706026 (unpublished).

${ }^{3}$ Y. Makhlin et al., Nature (London) 386, 305 (1999).

${ }^{4}$ V. Bouchiat et al., Phys. Scr., T 76, 165 (1998).

${ }^{5}$ Y. Nakamura et al., Nature (London) 398, 786 (1999).

${ }^{6}$ A. Aassime et al., Phys. Rev. Lett. 86, 3376 (2001).

${ }^{7}$ D. Vion et al., Science 296, 886 (2002).

${ }^{8}$ A. Shnirman and G. Schön, Phys. Rev. B 57, 15400 (1998).

${ }^{9}$ D.V. Averin, quant-ph/0008114 (unpublished).

${ }^{10}$ A. Cottet et al., in Proceedings of the MQC2 Conference, edited by D.V. Averin, B. Ruggiero, and P. Silvestrini (Kluwer/Plenum, New York, 2000).

${ }^{11}$ A. Korotkov, Appl. Phys. Lett. 69, 2593 (1996).

${ }^{12}$ D. Averin et al., Phys. Rev. Lett. 78, 4821 (1997).

${ }^{13}$ A. Käck et al., Phys. Rev. B 67, 035301 (2003).
${ }^{14}$ V. B. Braginsky and F. Y. Khalili, Quantum Measurement (Cambridge University Press, Cambridge, 1995).

${ }^{15}$ H. Schoeller and G. Schön, Phys. Rev. B 50, 18436 (1994).

${ }^{16}$ J. Spanier and K. B. Oldham, An Atlas of Functions (SpringerVerlag, Berlin, 1987).

${ }^{17}$ The Basset function of integer order $K_{n}$ is generally defined as an elliptic integral (Ref. 16)

$$
K_{v}(z)=\sqrt{\pi} /\left[\Gamma\left(\frac{1}{2}+v\right)\right](z / 2)^{v} \int_{1}^{\infty} e^{-z x} d x /\left(x^{2}-1\right)^{1 / 2-v} .
$$

The numerical analysis of the Basset function for small values of the argument $z$ is based on Neumann series of Bessel functions. For a large argument, a good algorithm can be written by applying the Padé technique on the asymptotic approximation for large $z K_{v}(z) \sim \sqrt{(\pi / 2 z)} e^{-z}\left[1+\sum_{j=1}^{\infty}\left[(v-1 / 2)_{j}(v\right.\right.$ $\left.\left.+1 / 2)_{j}\right] / 2 j z\right]$, where $(x)_{j}=x(x+1)(x+2) \cdots(x+j-1)$ is the Pochhammer polynomial.

${ }^{18}$ A.A. Clerk et al., Phys. Rev. Lett. 89, 176804 (2002). 